\title{
AC Losses of Prototype HTS Transmission Cables
}

\author{
J. W. Lue', J. A. Demko' ${ }^{1}$, L. Dresner ${ }^{2}$, R. L. Hughey ${ }^{3}$, U. Sinha ${ }^{3}$, J. C. Tolbert ${ }^{3}$, and S. K. Olsen ${ }^{4}$
}

1. Oak Ridge National Laboratory, Oak Ridge, TN, U. S. A.

2. Private Consultant, Oak Ridge, TN, U. S. A.

3. Southwire Co., Carrollton, GA, U. S. A.

4. Department of Electric Power Engineering, Technical University of Denmark, Denmark

Abstract - Since 1995 Southwire Company and Oak Ridge National Laboratory (ORNL) have jointly designed, built, and tested nine, 1-m long, high temperature superconducting (HTS) transmission cable prototypes. This paper summarizes the AC loss measurements of five of the cables not reported elsewhere, and compares the losses with each other and with theory developed by Dresner. Losses were measured with both a calorimetric and an electrical technique. Because of the broad resistive transition of the HTS tapes, the cables can be operated stably beyond their critical currents. The AC losses were measured in this region as well as below critical currents. Dresner's theory takes into account the broad resistive transition of the HTS tapes and calculates the AC losses both below and above the critical current. The two sets of $A C$ loss data agree with each other and with the theory quite well. In particular, at low currents of incomplete penetration, the loss data agree with the theoretical prediction of hysteresis loss based on only the outer two layers carrying the total current.

\section{INTRODUCTION}

Over the past three years Southwire Company and Oak Ridge National Laboratory (ORNL) have jointly designed, built, and tested nine, 1-m long, prototype high temperature superconducting (HTS) transmission cables. Complete test results of cable \#1, \#2, and \#5 were reported earlier [1], [2]. Cable \#7 was used to study the dependence of the number of layers on the performance of the cable by stripping the outermost layer after each test sequence. The results of that test will be reported separately [3]. A theory of calculating hysteresis loss of cables made of multi-layer of helically wound HTS tapes has been developed by Dresner [4]. This theory differs from others in that it accounts for the broad resistive transition of the HTS tapes and thus can calculate the $\mathrm{AC}$ loss both below and above the critical current of a cable. The latter is interesting because the HTS cables can be operated stably above their critical currents, due to the broad resistive transition. This paper summarizes the $A C$ loss measurements of five of the prototypes and compares the losses with each other and with Dresner's theory.

Manuscript received September 14, 1998

Research performed under a cooperative agreement between Southwire and Oak Ridge National Laboratory, the latter is managed by Lockheed Martin Energy Research Corp. for the U.S. Department of Energy under contract number DE-AC05-96OR22464.
TABLE I PROTOTYPE CABLE PARAMETERS

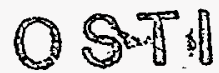

\begin{tabular}{l|ccccc}
\hline Cable Number & $\# 3$ & $\# 4$ & $\# 5 i$ & $\# 8$ & $\sharp 9$ \\
\hline No. of Layers & 10 & 4 & 2 & 4 & 4 \\
No. of Tapes & 200 & 112 & 48 & 104 & 100 \\
Cable ID (mm) & 25.7 & 38.4 & 38.2 & 38.4 & 38.2 \\
Cable OD (mm) & 35.1 & 41.7 & 40.4 & 41.4 & 40.1 \\
Lay angle ( $)$ & 15 & 15 & 30 & 28 & 28 \\
Lay pattern & +-+-+-+ & +-+- & +- & +-+- & ++-- \\
& +- & & & & \\
Cable $I_{c}^{*}(\mathrm{~A})$ & 1630 & 975 & 895 & 1605 & 1267 \\
& 1050 & 600 & 864 & 1465 & 1128 \\
n- value & 3.5 & 1.5 & 5 & 3.5 & 7 \\
\hline
\end{tabular}

* The two sets of $I_{c}^{\prime}$ 's were the results measured at two test sequences.

\section{DIFFERENCES IN THE CABLES}

Table I lists the parameters of the prototype cables. All cables were wound of $\mathrm{Bi}-2223 / \mathrm{Ag}$ HTS tapes made by Intermagnetic General Corp. (IGC) of about $3.5-\mathrm{mm}$ wide by $0.2-\mathrm{mm}$ thick. The tapes were helically wound on a stainless steel former with lay angles of about $15^{\circ}$ or $30^{\circ}$ as shown in the table. The lay angles were about the same for the different layers of each cable. Most cables were wound with an alternating pattern of left and right senses, except Cable \#9 which was wound with left-left and right-right senses. Kapton insulating tapes were used between neighboring layers of the cables, except Cables \#8 and \#9 where the insulating tapes were used only between layer \#2 and layer \#3. Cable \#3 had 10 layers of tapes, the rest had 4 layers. Cable \#3 was wound on a $2.54-\mathrm{cm}$-diameter former, the rest were wound on 38.1-cm-diameter formers. Cable \#5i designates the remaining 2-layer cable after the outer two layers were stripped off the original Cable \#5.

\section{AC LOSSMEASURING TECHNIQUES}

Two different techniques were used in measuring the $A C$ loss of the cables. The prototype cables were cooled in a liquid nitrogen bath at $77 \mathrm{~K}$. They were powered by a current transformer. Line frequency $60 \mathrm{~Hz} \mathrm{AC}$ currents of up to $2 \mathrm{kA}$ rms were used to charge the cables.

\section{A. Calorimetric Measurement}

Initially, all $\mathrm{AC}$ losses of the cables were measured with a calorimetric technique [5]. In this approach, the cable was 


\section{DISCLAIMER}

Portions of this document may be illegible in electronic image products. Images are produced from the best available original document. 
thermally insulated from the bath with a Micarta or G-10 tube filled with wax. A radial temperature gradient was developed by the $A C$ loss induced temperature rise of the conductor. The temperature rise, $\Delta \mathrm{T}$ on the surface of the cable relative to the liquid nitrogen bath was measured by thermocouples. The calibration of temperature rise versus loss rate was done with DC currents through the cable itself at values higher than the $I_{c}$ of the cable [1]. The product of the voltage drop and the current gave the power for the measured temperature rise. This technique is straight-forward but time consuming. At the low-loss range, it is also limited by the sensitivity of the thermocouples used (about $10 \mathrm{mK}$ in our setup). Depending on how well the cable was insulated, each test got a different $\Delta \mathrm{T}$ vs. loss rate curve and a maximum $\Delta \mathrm{T}$ before which the cable would overheat. Overall, $A C$ loss rates on the order of $0.01 \mathrm{~W} / \mathrm{m}$ to $10 \mathrm{~W} / \mathrm{m}$ were measured.

\section{B. Electrical Measurement}

More recently, an electrical technique was also used to measure the $\mathrm{AC}$ loss of most of the prototype cables. The voltage across the cable, and the phase-angle between the voltage and the current signals were measured by a digital lock-in amplifier. The product of the current and the voltage component in phase with the current gave the AC loss ( $P=$ I. $V \operatorname{Cos} \theta)$. This measurement can be done much quicker and the range of measurement can be very wide. However, when the loss voltage is small compared with the inductive voltage of the cable, the phase angle is near $90^{\circ}$. A slight error in the phase-angle measurement can lead to a big error (because of the cosine) in the loss result. Thus, it is important to correct any phase error that may be introduced from the reference signal relative to the true current phase. The location of the voltage tap and how the lead wires are routed can also give false results. For example, in one test setup, routing the voltage lead through the interior of the cable former resulted in a peaking out of the $A C$ loss and lower loss at higher currents that did not make sense.

The electrical measurements of the AC losses were performed months after all the cables were measured calorimetrically. The G-10 tube and the wax were removed from the cable for the electrical measurement. Different voltage tap locations were used in the two measurements. Different DC critical currents, $I_{c}$ were measured. Both sets of $I_{c}$ are listed in Table I. The lower $I_{c}$ values from the later measurements could be due to damage in removing the tube and wax, thermal degradation, or different voltage tap locations. The more than $30 \%$ decrease for Cable \#3 and \#4 had particular reasons. The current lead connections for Cable \#3 were made by clamping on the solder joint themselves while they were on $\mathrm{Cu}$-plugs for the rest of the cables. Thus, more damage in the lead sections were conceivable for Cable \#3. For Cable \#4, tape damages were visible on the cable. This was also reflected in the particularly low $n$-value of this cable. On the other hand, the resistive transition $n$-values were not changed much in the two measurements for most of the cables. Hence, average $n$ values are listed in the above table.

\section{SUMMARY OF DRESNER'S LOSS THEORY OF HTS CABLES}

\section{A. Hysteresis Losses in Power Law Cryoconductors}

The HTS tapes have a broad resistive transition, i.e.their resistivities increase gradually from unmeasurably small values at low currents to high values at high currents. Therefore, the critical-state theory (which assumes a sharp transition at critical current) of hysteresis loss developed for low temperature superconductors needs to be modified. The measured curves of resistivity, $\rho$ of HTS versus current density, $J$ can be reasonably well fitted by a power law: $\rho=$ $k \cdot J$. Using this power law, Dresner [6] calculated hysteresis losses in slab superconductors. (The slab assumption was based on the fact that HTS tapes generally have large aspect ratios ( 20$)$ ). By a combination of dimensional and numerical technique, Dresner derived formulas for the timeand space-averaged hysteresis power density, $Q\left(\mathrm{~W} / \mathrm{m}^{3}\right)$ for losses driven by either a sinusoidal transport current or an external field. For a peak transport current density of $J_{p}$, Dresner found:

$Q=G(n, \beta) ? b^{-1} ?\left(v \mu_{0} / J_{c}\right)^{(n+1) /(n+2)} ? E_{c}^{1 /(n+2)} ?\left(J_{p} b\right)^{\kappa}$.

where $\beta=J_{p} / J_{c}, \kappa=(3 n+4) /(n+2), b$ is the tape thickness, $v$ is the frequency, $\mu_{o}$ is the magnetic permittivity of free space $\left(4 \pi \times 10^{-7} \mathrm{Henry} / \mathrm{m}\right), E_{c}$ is the criteria for the critical current. $E_{c}$ can be $1 \mu \mathrm{V} / \mathrm{cm}$ or any other reasonable value as long as the corresponding critical current density, $J_{c}$ is used. Two asymptotic curves were found in that study.

In the limit of incomplete penetration, $\beta<1$

$G(n, \beta) \cup 1 / 6+017 ? n^{-0427}$.

Note that in this limit, $Q$ is proportional to $J_{p}$ to the $(3 n+4) /(n+2)$-power. In the case of very large $n$-value, this power equals the critical-state model value of 3 . Even for the lowest $n$-value (1.5) of our cables, this power is calculated to be 2.4 - not too much smaller.

In the limit of extreme full penetration, $\beta>>1$

$G(n, \beta)=D(n) ? \beta^{n(n+1) /(n+2)} ?\left(E_{c} /\left[v \mu_{0} J_{c} b^{2}\right]\right)^{(n+1) /(n+2)}$.

where $D(n)=\pi^{-05} \Gamma[(n+3) / 2] / \Gamma[(n+4) / 2]$, and $\Gamma$ is the gamma function. In this limit, $Q$ is proportional to $J_{p}$ to the $(n+2)$-power. This is understandable from the $f_{p}$ resistive power loss where $\rho$ is proportional to $f$. 


\section{B. Current Distribution in Multi-Layer Helical Cables}

Most HTS transmission cables consist of several layers of tapes wound helically on a hollow cylindrical former. Since the layers are in parallel, the question arises as to how the current distributes within and among them. Dresner considered the case where there are an even number of layers with adjacent layers having the same number of tapes, the same winding angle, but opposite sense of winding.

In the limiting case of incomplete penetration in a twolayer cable, the cable current is distributed in thin sheaths near the surfaces of each layer. In the outer layer, a current of $I \cdot \cos ^{2} \Theta$ is flowing in an outer sheath and of $I \cdot\left(\sin ^{2} \Theta-\cos ^{2} \Theta\right)$ 12 is flowing in an inner sheath $(\Theta$ is the lay angle). In the inner layer, there is a current of $I / 2$ flowing in the outer sheath and no current is flowing in the inner sheath. An important consequence of this result is that under the above assumptions there is no magnetic field at all within the inner layer or in the bore of the former. Thus, in the limit of incomplete penetration (of the outer two layers), only the outer two layers carry current, even when there are more than two layers. Compare the helical cable with the case where all the tapes are isolated from one another and share the current equally, one needs to multiply the above current distribution factor to the loss for the cable. In terms of hysteresis power loss per unit length of a cable, $P_{\text {cable }}$ was found in the limit of incomplete penetration to be

$$
P_{c a b l e}=2 \pi D b ? 4^{\kappa-1}\left[\cos ^{2 \kappa} \Theta+\left(\left|\sin ^{2} \Theta-\cos ^{2} \Theta\right| / 2\right)^{\kappa}+2^{-\kappa}\right] ? Q,
$$

where $D$ is the diameter of the cable.

In the limiting case of full penetration, the current is equally shared among the tapes so that there is no difference from the situation in which all the tapes of the cable are isolated from one another. In this limit, the power loss per unit length was found to be

$$
P_{\text {cable }}=L \pi D b ? Q \text {. }
$$

where $L$ is the number of layers. Note also that when converting the rms or peak current density to cable current one needs to use the surface area of the tapes of the outer two layers $(2 \mathrm{Nab})$ in the limit of incomplete penetration and the total surface area of the cable ( $L N a b)$ in the limit of full penetration, where $N$ is the number of tapes per layer, and $a$ is the width of a tape.

\section{COMPARISON OF DIFFERENT MEASUREMENT RESULTS}

The calorimetrically and electrically measured $A C$ losses of Cable \#3 as a function of rms currents are shown in Fig. 1 together with Dresner's theoretical calculations. It is seen that the two sets of data agree with each other reasonably

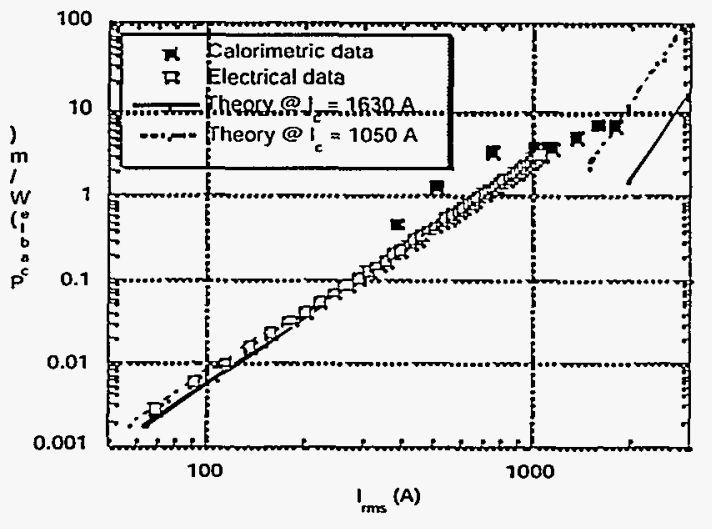

Fig. 1 ACloss of Cable $\# 3$ measured by two different techniques.

well over the range where the data overlap. As was noted earlier, the electrical measuring technique was more sensitive and data was extended to a far lower loss range. The agreement of the data with Dresner's incomplete penetration asymptote is surprisingly good. Note that Dresner's theory predicted that (for the situation considered) only the outer two layers carry currents in this limit. The data fully confirmed this prediction. Note that the two incomplete penetration asymptotes based on the two different measured $I_{c}$ 's were drawn to currents where $I_{p}$ equaled about $90 \%$ of the $I_{c}$ of the outer two layers. It is interesting to note that the data seemed to follow the same trend beyond these currents.

Fig. 2 shows similar comparison curves for Cable \#9. This cable was built to simulate a 2-layer winding of a 4layer cable. Namely, the layers were wound with a left-leftright-right pattern. The theoretical curves in Fig. 2 were calculated based on a 2-layer cable with double-tape thickness, $2 b$. The agreement between the two sets of data is even better. They also agree with the theoretical curves quite well. The two-

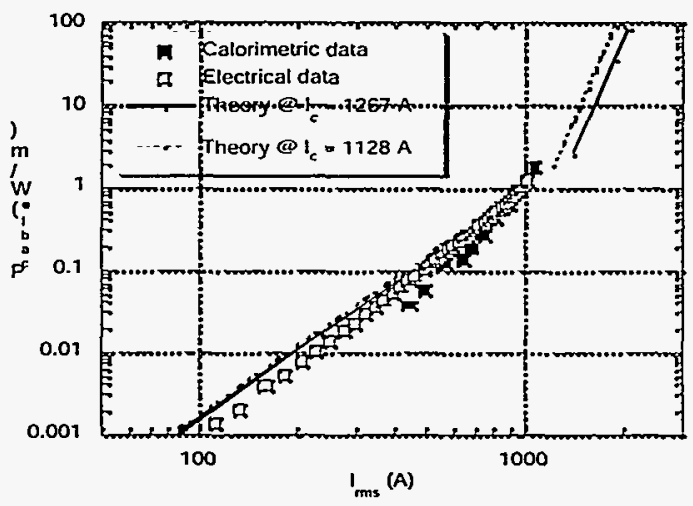

Fig. 2 ACloss of Cable \#9 measured by two different techniques. 